\title{
Adaptive Robust Actuator Fault Accommodation for a Class of Uncertain Nonlinear Systems with Unknown Control Gains
}

\author{
Yuefei Wu, ${ }^{1}$ Dawei Ma, ${ }^{1}$ Jianyong Yao, ${ }^{1}$ Guigao Le, ${ }^{1}$ Zhenxing $\mathrm{Mu}^{2}$ and Fan Yang $^{3}$ \\ ${ }^{1}$ School of Mechanical Engineering, Nanjing University of Science and Technology, Nanjing 210094, China \\ ${ }^{2}$ North Navigation Technology Group Co., Beijing 100041, China \\ ${ }^{3}$ LTD Nanjing Aircraft Manufacturing Institute of NPV, Nanjing 210094, China
}

Correspondence should be addressed to Jianyong Yao; jerryyao.buaa@gmail.com

Received 21 November 2013; Accepted 30 December 2013; Published 8 April 2014

Academic Editor: Weichao Sun

Copyright (C) 2014 Yuefei Wu et al. This is an open access article distributed under the Creative Commons Attribution License, which permits unrestricted use, distribution, and reproduction in any medium, provided the original work is properly cited.

An adaptive robust fault tolerant control approach is proposed for a class of uncertain nonlinear systems with unknown signs of high-frequency gain and unmeasured states. In the recursive design, neural networks are employed to approximate the unknown nonlinear functions, K-filters are designed to estimate the unmeasured states, and a dynamical signal and Nussbaum gain functions are introduced to handle the unknown sign of the virtual control direction. By incorporating the switching function $\sigma$ algorithm, the adaptive backstepping scheme developed in this paper does not require the real value of the actuator failure. It is mathematically proved that the proposed adaptive robust fault tolerant control approach can guarantee that all the signals of the closed-loop system are bounded, and the output converges to a small neighborhood of the origin. The effectiveness of the proposed approach is illustrated by the simulation examples.

\section{Introduction}

In complex systems like chemical plants, nuclear reactors, and flight control systems, reliability is as important as performance. Conventional feedback design [1] for such complex systems may result in unacceptable degradation in performance or even instability in the event such as actuators, sensors, and processors that may undergo abrupt failures individually or simultaneously during operation. The adverse effects due to the failures require being compensated to enhance the reliability and safety of the system. The research on accommodating such failures and maintaining acceptable system performance is particularly important. System faults are typically characterized by critical changes in the system parameters and changes in the inherent dynamical structure of the system. Hence, effective fault diagnosis and accommodation (FDA) have become an important area of research [2$4]$.
In this work, we focus on the problem of actuator failure accommodation. Various approaches to FDA using analytical redundancy have been reported during the last three decades. Generally speaking, the control methods can be clarified into the following types: fault detection and diagnosis designs [5-7]; linear matrix inequality techniques $[8,9]$; adaptive approaches [10, 11]; and so forth. Among these design methods, adaptive mechanisms [12-16] have been employed, and adaptive control has been a promising approach to deal with such failures. In adaptive control systems, controllers were designed with the aid of adaptation mechanisms to handle large uncertain structural and parametric variation caused by failures. In [17], a novel attempt was made to compensate for the actuator failures in linear time-invariant systems by using adaptive state feedback. However, all states were assumed to be available for this proposed scheme. As noted in [18], in practice, state variables were often immeasurable for many practical nonlinear systems. In such cases, 
an adaptive output-feedback control scheme should be developed. In [19], an adaptive output-feedback controller was synthesized. Furthermore, nonlinear systems with actuator failures were investigated. In [20], adaptive state feedback failure compensation schemes were proposed for nonlinear systems in the parametric strict-feedback form. However, nonlinear behaviors [10, 21-23] and modeling uncertainties complicate the development of high-performance closedloop controllers. A robust adaptive state feedback failure compensation method considering modeling uncertainties was proposed in $[12,24,25]$. Apparently, such techniques were not well suited to suppress the undesirable transients when facing a sudden change in system parameters due to unknown actuator faults. In [26, 27], a robust modelbased fault detection scheme was developed by using adaptive robust strategy to deal with parametric uncertainties and bounded uncertainty nonlinearities.

Recently, the problem of adaptive control of systems with the unknown sign of high frequency gain has also received much attention. How to weaken the high frequency gain sign assumptions is an important issue. The Nussbaum-type function was originally proposed by [28] for dealing with unknown sign of high frequency gain. This method was then generalized to higher order linear systems by [29]. For a class of time-varying parameter high-order uncertain nonlinear systems, a robust adaptive output-feedback control method was proposed in [30-33] for the unknown control gain direction and unpredictable state. However, the proposed approaches were only focused on the so-called nonlinear strict-feedback systems, in which the nonlinear uncertainties were known or can be linearly parameterized. In [34], an adaptive neural network backstepping control scheme has been developed, an adaptive neural network backstepping control scheme for a given class of nonlinear systems, and neural network systems were used to approximate the unknown nonlinear functions, and the stability of the closedloop system was given based on iterative Lyapunov design. This result has been extended by [35] to a class of the nonlinear time-delay systems in the strict-feedback form. However, there is little work using this method to deal with unknown actuator failures and unknown control gain simultaneously.

In this paper, we propose an adaptive robust approach for actuator fault-tolerant control (ARFTC) of a class of uncertain nonlinear systems. Moreover, compared with [10, $12,24]$, a parameterisable time-varying actuator failure model is investigated. The technique here is a combination of adaptive backstepping [36] and switching function $\sigma$ algorithm based ARFTC proposed in [27] and differs significantly from the techniques presented in [19] which relies on backstepping based direct adaptive control. Specifically, ARFTC uses robust filter structures to attenuate the effect of model uncertainties, and adaptation is used only as a means to reduce the extent of parametric uncertainties. However, neither adaptive control nor robust control based fault-tolerant designs can address the issues associated with actuator faults. In the present work, we claim that an adaptive robust fault-tolerant control scheme integrates adaptive and robust control design techniques. In order to show the superior performance of the proposed scheme, comparative studies are performed using simulation examples.

\section{Problem Formulation and Preliminaries}

2.1. Problem Formulation. In this paper, we consider the following nonlinear system as [27]

$$
\begin{aligned}
\dot{x}_{1}= & x_{2}+f_{1}(y)+\varphi_{0,1}(y)+d_{1}, \\
& \vdots \\
\dot{x}_{\rho-1}= & x_{\rho}+f_{\rho-1}(y)+\varphi_{0, \rho-1}(y)+d_{\rho-1}(t, x), \\
\dot{x}_{\rho}= & x_{\rho+1}+f_{\rho}(y)+\varphi_{0, \rho}(y)+\sum_{j=2}^{\rho} f_{j, \rho}(y) x_{j} \\
& +\sum_{j=1}^{q} b_{m, j} \beta_{j}(y) u_{j}+d_{\rho}(t, x), \quad 2 \leq \rho \leq n-1, \\
& \vdots \\
\dot{x}_{n-1}= & x_{n-1}+f_{n-1}(y)+\varphi_{0, n-1}(y)+\sum_{j=2}^{n-1} f_{j, n-1}(y) x_{j} \\
& +\sum_{j=2}^{n} f_{j, n}(y) x_{j}+d_{n}(t, x), \quad y=x_{1}, \\
& +\sum_{j=1}^{q} b_{1, j} \beta_{j}(y) u_{j}+d_{n-1}(t, x), \\
\dot{x}_{n}= & \varphi_{0, n}(y)+f_{n}(y)+\sum_{j=1}^{q} b_{0, j} \beta_{j}(y) u_{j}
\end{aligned}
$$

where $u_{j} \in R, j=1,2, \ldots, q$ are the control inputs whose actuators may fail during operation; $x=\left[x_{1}, x_{2}, \ldots, x_{n}\right]^{T} \in$ $R^{n}$ is the state vector; $y \in R$ is the system output; $f_{i}(y), i=$ $1,2, \ldots, n$ are unknown smooth functions, which represent model uncertainties due to modeling errors or unmodeled dynamics; $d_{i}(t)$ are bounded time-varying disturbances with unknown constant bounds; $b_{i, j}, i=0,1, \ldots, m, j=1,2, \ldots, q$ are unknown constant parameters; $\varphi_{0, i}(y), i=1,2, \ldots, n$, $\beta_{j}(y), j=1,2, \ldots, q$ are known smooth nonlinear functions; $b_{i, j} \beta_{j}(y) \neq 0$ for $y \in R$. Only the output $y$ is available for measurement.

Assumption 1 (see [27]). System (1) is such that the desired control objective can be fulfilled with up to $m-1$ stuck actuators, the remaining actuators can still achieve a desired control objective when implemented with the knowledge of the plant parameters and failure parameters.

Assumption 2 (see [26]). The relative degree $\rho=n-m$ is known and the system is minimum phase; the polynomial $B(s)=b_{m} s_{m}+\cdots+b_{1} s+b_{0}$ is Hurwitz. 
Assumption 3. The unknown disturbance $d_{i}(t)$ satisfies $\left|d_{i}(t)\right| \leq d_{i}$, where $d_{i}$ is an unknown constant.

A time-varying actuator failure can be modeled as [27]

$$
u_{j}(t)=\bar{u}_{j}+\bar{d}_{j}(t), \quad t \geq t_{j}, j \in\{1,2, \ldots, q\},
$$

where the failure value $\bar{u}_{j}$, the failure time instant $t_{j}$, and the failure index $j$ are unknown; $\bar{d}_{j}(t)$ is given by

$$
\bar{d}_{j}(t)=\sum_{l=1}^{h} \bar{d}_{j l} f_{j l}(t)
$$

for some unknown scalar constants $\bar{d}_{j l}$ and known bounded scalar signals $f_{j l}(t), j=1,2, \ldots, q, l=1,2, \ldots, h, h \geq 1$.

Thus, the actuator failure mode is defined as [26]

$$
u_{j}(t)= \begin{cases}\bar{u}_{j}+\bar{d}_{j}(t), & \text { if stuck actuator, } t \geq T_{f} \\ \eta_{j} \nu_{j}(t), & \text { if loss of efficiency, } t \geq T_{f},\end{cases}
$$

where $v_{j}(t), j=1,2, \ldots, q$ are applied control signals from a feedback control design, and where $T_{f}$ is the unknown instant of failure, $\bar{u}_{j}$ is an unknown constant value at which the actuator gets stuck, and $\eta_{j} \in\left[\left(\eta_{j}\right)_{\min }, 1\right]$ represents actuator loss in efficiency.

The control target is that all the closed-loop signals remain bounded, while the plant output $y(t)$ asymptotically tracks a prescribed signal $y_{d}(t)$ despite the presence of unknown actuator failures, unknown plant parameters, and unknown control gain signs. The reference signal $y_{d}(t)$ and its derivatives are known and bounded.

2.2. Nussbaum Function Properties. To deal with the unknown control gain signs, we introduce the knowledge of Nussbaum-type gain. A smooth function $N(k): R \rightarrow R$ is called Nussbaum-type gain if it has the following properties [29]

$$
\begin{aligned}
& \lim _{s \rightarrow+\infty} \sup \frac{1}{s} \int_{0}^{s} N(\varsigma) d \varsigma=+\infty, \\
& \lim _{s \rightarrow+\infty} \inf \frac{1}{s} \int_{0}^{s} N(\varsigma) d \varsigma=-\infty .
\end{aligned}
$$

For instance, $k^{2} \cos (k)$ and $k^{2} \sin (k)$ belong to this class of functions. In this paper, an even Nussbaum-type function $k^{2} \cos (k)$ is used.

Lemma 4 (see [35]). Let $V(\cdot)$ and $k(\cdot)$ be smooth functions defined on $\left[0, t_{f}\right)$ with $V(t) \geq 0, t \in\left[0, t_{f}\right)$, and $N(\cdot)$ are an even smooth Nussbaum-type function. If the following inequality holds

$$
V(t) \leq c_{0}+e^{-c_{1} \tau} \int_{0}^{t}[x(\tau) N(k)+1] \dot{k} e^{c_{1} \tau} d \tau,
$$

where $c_{0}$ represents a suitable constant, $c_{1}$ is a positive constant, and $x(t)$ is a time-varying parameter taking values in the unknown closed intervals, then $V(t), k(t), \int_{0}^{t} x(\tau) N(k) \dot{k} d \tau$ must be bounded on $\left[0, t_{f}\right)$.
2.3. Neural Networks (NNs). NNs have been widely used in modeling and control of nonlinear systems due to their good capabilities of nonlinear function approximation, learning, and fault tolerance [34]. The following radial basis function NNs (RBFNNs) are used to approximate the continuous function $f_{i}(y): R \rightarrow R:$

$$
f_{i}(y)=\mathbf{W}_{i}^{T} \boldsymbol{\Phi}_{i}(y)
$$

where the input $y \in R$; the weight vector $\Phi_{i}=\left[\Phi_{i 1}, \ldots, \Phi_{i l}\right]^{T}$, $\mathbf{W}_{i}=\left[\omega_{i 1}, \ldots, \omega_{i l}\right]^{T}$ with the NN node number $l$; the vector of smooth basis functions $\Phi_{i j}$ being chosen as the commonly used Gaussian functions $\boldsymbol{\Phi}_{i}=\exp \left(-\left(y-\mathrm{c}_{i}\right)^{2} /\left(\eta_{i}^{2}\right)\right), i=$ $1,2, \ldots, l$, where $\mathbf{c}_{i}=\left[c_{i 1}, \ldots, c_{i l}\right]^{T}$ is the center of the receptive field and $\eta_{i}$ is the width of the Gaussian function. It has been proven in [34] that networks (7) can approximate any smooth functions over a compact set $y \in R$ to accuracy as

$$
f_{i}(y)=\mathbf{W}_{i}^{* T} \boldsymbol{\Phi}_{i}(y)+\varepsilon_{i}
$$

where $\mathbf{W}_{i}^{*}$ is ideal constant weights, and the approximation error $\varepsilon_{i}(y)$ satisfies $\left|\varepsilon_{i}(y)\right| \leq \varepsilon_{i}$ with constant $\varepsilon_{i}>0$.

The ideal weight vector $\mathbf{W}_{i}^{*}$ is defined as the value of $\mathbf{W}_{i}^{*}$ that minimizes $\left|\varepsilon_{i}(y)\right|$; that is,

$$
\mathbf{W}_{i}^{*}=\arg \min _{\mathbf{W}_{i}}\left\{\sup _{y \in \Omega_{y}}\left|f_{i}(y)-\widehat{\mathbf{W}}_{i}^{T} \boldsymbol{\Phi}_{i}(y)\right|\right\} .
$$

\section{Output-Feedback Based ARFTC}

3.1. State Estimation. Control signals $u_{j}(t)$ are designed such that $u^{*}=u_{j} \beta(y)$. With fault model (2) and the chosen actuation scheme, we can rewrite the control inputs as follows:

$$
u_{j}(t)=\frac{\eta_{j}}{\beta_{j}(y)}\left(1-\sigma_{j}\right) u^{*}(t)+\sigma_{j} \bar{u}_{j}, \quad j=1, \ldots, q,
$$

where $\sigma_{j}=1$ corresponds to stuck actuator, and $\sigma_{j}=0$, $\eta_{j} \in\left[\left(\eta_{j}\right)_{\min }, 1\right]$ represents efficiency loss of the actuator. In accordance with this, we rewrite the system as follows:

$$
\begin{gathered}
\dot{x}=\mathbf{A} x+\varphi_{0}(y)+\mathbf{W}^{* T} \boldsymbol{\Phi}(y)+\mathbf{F}(y) x+\sum_{i=0}^{m} \mathbf{e}_{n-i} \kappa_{i} u^{*} \\
+\sum_{j=1}^{q} \sum_{i=0}^{m} \mathbf{e}_{n-i} \mu_{i, j} \boldsymbol{\beta}_{j}(y)+\Delta(y, t), \\
y=\mathbf{c}^{T} \mathbf{x},
\end{gathered}
$$


where $e_{1}$ denotes the first coordinate vector in $R^{n}$

$$
\begin{aligned}
& \mathbf{A}=\left[\begin{array}{lll}
0 & & \\
\vdots & I_{n-1} & \\
0 & & 0
\end{array}\right], \\
& \Phi(y)=\left[\begin{array}{cccc}
\Phi_{1}(y) & 0 & \cdots & 0 \\
0 & \Phi_{2}(y) & \ddots & \vdots \\
\vdots & \vdots & \ddots & 0 \\
0 & 0 & \cdots & \Phi_{n}(y)
\end{array}\right] \text {, } \\
& \mathbf{F}(y)=\left[\begin{array}{cccc}
0 & 0 & \cdots & 0 \\
0 & f_{2,2} & \ddots & \vdots \\
\vdots & \vdots & \ddots & 0 \\
0 & f_{2,2} & \cdots & f_{n, n}
\end{array}\right] \text {, } \\
& \varphi_{0}(y)=\left[\begin{array}{c}
\varphi_{0,1}(y) \\
\varphi_{0,2}(y) \\
\vdots \\
\varphi_{0, n}(y)
\end{array}\right], \quad \Delta=\left[\begin{array}{c}
d_{1}+\varepsilon_{1} \\
d_{2}+\varepsilon_{2} \\
\vdots \\
d_{n}+\varepsilon_{n}
\end{array}\right] \text {, } \\
& \kappa_{i}=\sum_{j=1}^{q} \eta_{j}\left(1-\sigma_{j}\right) b_{i, j}, \quad \mu_{i, j}=\sigma_{j} \bar{u}_{j} b_{i, j}, \\
& \mathbf{c}=\left[\begin{array}{llll}
1 & 0 & \cdots & 0
\end{array}\right]^{T}, \quad \mathbf{W}^{*}=\left[\begin{array}{llll}
\mathbf{W}_{1}^{*} & \mathbf{W}_{2}^{*} & \cdots & \mathbf{W}_{n}^{*}
\end{array}\right],
\end{aligned}
$$

where $i=0,1, \ldots, m, j=1,2, \ldots, q$. It can be deduced from Assumption 3 that $|\Delta(x, t)| \leq \varepsilon_{i}+d_{i}<\psi_{m}$, where $\psi_{m}$ is an unknown bounded constant.

Note that $\kappa_{i}$ is the unknown measure of actuator effectiveness after faults and $\mu_{i, j}$ is the unknown measure of the fault magnitude which needs to be compensated.

Thus, the states of system (1), unknown constants and parameter vectors, should be estimated by using the filters given in $[26,27]$. We will define the following set of filters for the purpose of state-estimation:

$$
\begin{gathered}
\dot{\boldsymbol{\xi}}_{0}=\left(\mathbf{A}-l \mathbf{L} \mathbf{\mathbf { q c }}{ }^{T}\right) \boldsymbol{\xi}_{0}+l \mathbf{L} \mathbf{q} y+\varphi_{0}(y)+\mathbf{F}(y) \boldsymbol{\xi}_{0}, \quad \boldsymbol{\xi}_{0} \in R^{n \times 1} \\
\dot{\boldsymbol{\xi}}=\left(\mathbf{A}-l \mathbf{L} \mathbf{q} c^{T}\right) \boldsymbol{\xi}+\Phi(y), \quad \xi \in R^{n \times l n} \\
\dot{\boldsymbol{v}}_{i}=\left(\mathbf{A}-l \mathbf{L} \mathbf{q c ^ { T }}\right) \boldsymbol{\nu}_{i}+\mathbf{e}_{n-i} u^{*}+\mathbf{F}(y) \boldsymbol{v}_{i}, \quad \boldsymbol{\nu}_{i} \in R^{n \times 1} \\
\dot{\psi}_{i, j}=\left(\mathbf{A}-l \mathbf{L} \mathbf{q} c^{T}\right) \psi_{i, j}+\mathbf{e}_{n-i} \boldsymbol{\beta}_{j}(y), \quad \psi_{i, j} \in R^{n \times 1},
\end{gathered}
$$

where the gain matrix $\mathbf{q}=\left[q_{1}, q_{2}, \ldots, q_{n}\right]^{T}$ is chosen to make $\mathbf{A}-\mathbf{q c}^{T}$ Hurwitz, $i=0,1, \ldots, m, j=1,2, \ldots, q$, $\mathbf{L}=\operatorname{diag}\left[\begin{array}{llll}1 & l & \cdots & l^{n-1}\end{array}\right]$, and $l$ is the observer gain updated by

$$
\dot{l}=-\kappa l^{2}+\kappa l+l \gamma(y), \quad l(0)=1
$$

with $\kappa$ a positive design parameter and $\gamma(y)$ a nonnegative smooth function. It can be proved by contradiction that $l(t) \geq$ 1 for all $t \geq 0$.
Due to the special structure of $\mathbf{A}$, the order of K-filters can be reduced by using the following two filters:

$$
\begin{gathered}
\dot{\lambda}=\left(\mathbf{A}-l \mathbf{L} \mathbf{q} c^{T}\right) \lambda+\mathbf{e}_{n} u^{*}+\mathbf{F}(y) \lambda, \\
\dot{\boldsymbol{\zeta}}_{j}=\left(\mathbf{A}-l \mathbf{L} \mathbf{q} c^{T}\right) \boldsymbol{c}_{j}+\mathbf{e}_{n} \boldsymbol{\beta}_{j}(y), \quad j=1,2, \ldots, q
\end{gathered}
$$

and the following algebraic equations:

$$
\begin{gathered}
\boldsymbol{\nu}_{i}=\left(\mathbf{A}-l \mathbf{L} \mathbf{q} \mathbf{c}^{T}\right)^{i} \lambda, \\
\boldsymbol{\psi}_{i, j}=\left(\mathbf{A}-l \mathbf{L} \mathbf{q} c^{T}\right)^{i} \boldsymbol{s}_{j}, \quad i=0,1, \ldots, m .
\end{gathered}
$$

The estimated state can be written as

$$
\widehat{\mathbf{x}}=\boldsymbol{\xi}_{0}+\boldsymbol{\xi} \mathbf{W}^{*}+\sum_{i=0}^{m} \kappa_{i} \boldsymbol{\nu}_{i}+\sum_{j=1}^{q} \sum_{i=0}^{m} \mu_{i, j} \boldsymbol{\psi}_{i, j}
$$

Let $\widetilde{\mathbf{x}}=\mathbf{x}-\widehat{\mathbf{x}}$ be the estimation error. Then, the state estimation error dynamic is given by

$$
\dot{\tilde{\mathbf{x}}}(t)=\left(\mathbf{A}-l \mathbf{L} \mathbf{q} \mathbf{c}^{T}\right) \widetilde{\mathbf{x}}+\mathbf{F}(y) \widetilde{\mathbf{x}}+\Delta .
$$

Noting that the change of coordinates $\overline{\mathbf{x}}=l^{-\mu} \mathbf{L}^{-1} \widetilde{\mathbf{X}}$ with $\mu$ a positive design parameter (18) is transformed into

$$
\dot{\overline{\mathbf{x}}}=l\left(\mathbf{A}-\mathbf{q} c^{T}\right) \overline{\mathbf{x}}+\mathbf{L}^{-1} \mathbf{F}(y) \mathbf{L} \overline{\mathbf{x}}+l^{-\mu} \mathbf{L}^{-1} \boldsymbol{\Delta}-\frac{\dot{l}}{l}(\mu \mathbf{I}+\mathbf{D}) \overline{\mathbf{x}},
$$

where $D=\operatorname{diag}\{0,1, \ldots, n-1\}$. Since $\mathbf{A}-\mathbf{q c}^{T}$ is Hurwitz, there is a symmetric positive definite matrix $\mathbf{P}$ satisfying $\mathbf{P}\left(\mathbf{A}-\mathbf{q} \mathbf{c}^{T}\right)+\left(\mathbf{A}-\mathbf{q} \mathbf{c}^{T}\right)^{T} \mathbf{P}=-\mathbf{I}$. Let the quadratic Lyapunov function $V_{x}=\overline{\mathbf{x}}^{T} \mathbf{P} \overline{\mathbf{x}}$, whose derivative is computed as

$$
\begin{aligned}
\dot{V}_{x}= & -l\|\overline{\mathbf{x}}\|^{2}+2 \overline{\mathbf{x}}^{T} \mathbf{P} \mathbf{L}^{-1} \mathbf{F}(y) \mathbf{L} \overline{\mathbf{x}}+2 l^{-\mu} \overline{\mathbf{x}}^{T} \mathbf{P} \mathbf{L}^{-1} \boldsymbol{\Delta} \\
& -\frac{i}{l} \overline{\mathbf{x}}^{T}(\mathbf{P D}+\mathbf{D P}+2 \mu \mathbf{P}) \overline{\mathbf{x}} .
\end{aligned}
$$

Note that $l \geq 1$. Then there is a nonnegative smooth function $\gamma_{1}(y)$ such that $\mathbf{L}^{-1} \mathbf{F}(y) \mathbf{L} \leq \gamma_{1}(y)$, from which it follows that

$$
2 \overline{\mathbf{x}}^{T} \mathbf{P} \mathbf{L}^{-1} \mathbf{F}(y) \mathbf{L} \overline{\mathbf{x}} \leq 2\|\mathbf{P}\| \gamma_{1}(y)\|\overline{\mathbf{x}}\|^{2} .
$$

From Young's inequality, we have

$$
2 l^{-\mu} \overline{\mathbf{x}}^{T} \mathbf{P L}^{-1} \Delta \leq l^{-2 \mu} \overline{\mathbf{x}}^{T} \overline{\mathbf{x}}+\left\|\mathbf{P} \mathbf{L}^{-1}\right\|^{2} \psi_{m}^{2} .
$$

Since $\mathbf{P}$ is a symmetric positive definite matrix, by choosing a sufficiently large $\mu$, we can obtain

$$
\sigma_{1} \mathbf{I} \leq \mathbf{D P}+\mathbf{P D}+2 \mu \mathbf{P} \leq \sigma_{2} \mathbf{I}
$$

which together with (14) implies that

$$
-\frac{i}{l} \overline{\mathbf{x}}^{T}(\mathbf{P D}+\mathbf{D P}+\mathbf{2} \mu \mathbf{P}) \overline{\mathbf{x}} \leq \kappa \sigma_{2} l\|\overline{\mathbf{x}}\|^{2}-\sigma_{1} \gamma(y)\|\overline{\mathbf{x}}\|^{2},
$$


where $\sigma_{1}, \sigma_{2}$ are positive constants. From (21) and (24), (18) can be rewritten as

$$
\begin{aligned}
\dot{V}_{x} \leq-\left[\left(1-l^{-2 \mu}-\kappa \sigma_{2}\right) l+\sigma_{1} \gamma(y)\right. & \\
& \left.-2\|\mathbf{P}\| \gamma_{1}(y)\right]\|\overline{\mathbf{x}}\|^{2}+\left\|\mathbf{P} \mathbf{L}^{-1}\right\|^{2} \psi_{m}^{2} .
\end{aligned}
$$

By choosing $\kappa$ and $\gamma(y)$ to satisfy $\kappa \leq\left(1-2 l^{-2 \mu}\right) /\left(2 \sigma_{2}\right), \gamma(y) \geq$ $2\|P\| \gamma_{1}(y) / \sigma_{1} \geq 0$, we arrive at

$$
\dot{V}_{x} \leq-\frac{1}{2} l\|\overline{\mathbf{x}}\|^{2}+\left\|\mathbf{P L}^{-1}\right\|^{2} \psi_{m}^{2}
$$

3.2. Parameter Estimate. Let $\widehat{\boldsymbol{\theta}}$ denote the estimate of $\boldsymbol{\theta}$ and $\widetilde{\boldsymbol{\theta}}=\boldsymbol{\theta}-\widehat{\boldsymbol{\theta}}$ denote the estimation error. The extent of parametric uncertainties satisfy

$$
\Omega=\left\{\boldsymbol{\theta} \mid\|\boldsymbol{\theta}\| \leq M_{1}\right\},
$$

where $M_{1}$ is a positive design parameter.

It is well known that parameter estimation algorithms suffer from parameter drift in presence of disturbances, resulting in system states growing unboundedly. We use the switching function $\sigma$ algorithm [36] to deal with this problem. The update law used here has the following form:

$$
\sigma_{\theta}(\|\hat{\boldsymbol{\theta}}\|)= \begin{cases}0, & \|\hat{\boldsymbol{\theta}}\| \leq M_{1} \\ \chi_{1}(\|\hat{\boldsymbol{\theta}}\|), & M_{1} \leq\|\hat{\boldsymbol{\theta}}\| \leq 2 M_{1} \\ \sigma_{10}, & \|\hat{\boldsymbol{\theta}}\| \geq 2 M_{1},\end{cases}
$$

where $\sigma_{10}$ is a positive design parameter, and $\chi_{1}$ is an arbitrary adaptation function.

Consider

$$
\chi_{1}(\|\hat{\boldsymbol{\theta}}\|)=\frac{\sigma_{10}(2 \rho-1) !}{M_{1}^{2 \rho-1}[(\rho-1) !]^{2}} \int_{M_{1}}^{\|\hat{\boldsymbol{\theta}}\|}\left(\Theta-M_{1}\right)\left(2 M_{1}-\Theta\right) d \Theta
$$

The mapping guarantees that the following properties are always satisfied:

$$
\sigma_{\theta} \widetilde{\boldsymbol{\theta}}^{T} \widehat{\boldsymbol{\theta}} \leq-\frac{1}{2} \sigma_{10} \widetilde{\boldsymbol{\theta}}^{T} \widetilde{\boldsymbol{\theta}}+\frac{13}{2} \sigma_{10} M_{1}^{2}
$$

3.3. Controller Design. Furthermore, system (13) can be represented as

$$
\begin{gathered}
\dot{v}_{m, i}=\dot{v}_{m, i+1}-q_{i} l^{i} \dot{v}_{m, 1}, \quad i=2,3, \ldots, \rho-1, \\
\dot{v}_{m, \rho}=\dot{v}_{m, \rho+1}-q_{\rho} l^{\rho} \dot{v}_{m, 1}+u^{*} .
\end{gathered}
$$

The derivative of the output $y$ is given by

$$
\begin{aligned}
\dot{y} & =\boldsymbol{\omega}_{0}+\boldsymbol{\omega}^{T} \boldsymbol{\theta}+\Delta_{1}(y, t) \\
& =\kappa_{m} v_{m, 2}+\boldsymbol{\omega}_{0}+\overline{\boldsymbol{\omega}}^{T} \boldsymbol{\theta}+\Delta_{1}(y, t),
\end{aligned}
$$

where the regressor $\boldsymbol{\omega}$ and truncated regressor $\boldsymbol{\omega}_{0}$ are defined as [26]

$$
\begin{gathered}
\boldsymbol{\omega}_{0}=\left[\xi_{0,2}+\varphi_{0,1}\right] \\
\boldsymbol{\omega}=\left[\boldsymbol{\xi}_{(2)}+\boldsymbol{\Phi}_{(1)}, v_{m, 2}, v_{m-1,2}, \ldots, v_{0,2},\right. \\
\left.\psi_{m, 1(2)}, \ldots, \psi_{m, q(2)}, \ldots, \psi_{0,1(2)}, \ldots, \psi_{0, q(2)},\right]^{T}, \\
\overline{\boldsymbol{\omega}}=\boldsymbol{\omega}-e_{l n+1} v_{m, 2} \\
\boldsymbol{\theta}=\left[\mathbf{W}^{*}, \kappa_{m}, \ldots, \kappa_{0}, \mu_{m, 1}, \ldots, \mu_{m, q}, \ldots, \mu_{0,1}, \ldots, \mu_{0, q}\right]^{T} .
\end{gathered}
$$

In this section, we present the adaptive output-feedback control design using the backstepping technique. Define the following error coordinates: $z_{1}=y-y_{d}$ and $z_{i}=v_{m, i}-\alpha_{i-1}$, $i=2,3, \ldots, \rho$, where $\alpha_{i-1}$ is the stabilizing functions to be designed.

Step 1. Differentiating $z_{1}$ with respect to time $t$, we obtain

$$
\dot{z}_{1}=\kappa_{m} v_{m, 2}+\boldsymbol{\omega}_{0}+\overline{\boldsymbol{\omega}}^{T} \boldsymbol{\theta}-\dot{y}_{d}+\Delta_{1}(y, t) .
$$

The problem of the unknown sign of the virtual direction is sloved by the Nussbaum-type functions $\kappa_{m}$. Choose the tuning functions and parameter adaptation law as

$$
\begin{gathered}
\alpha_{1}=N(k) \tau, \\
\dot{k}=z_{1} \tau, \\
\tau=c_{1} z_{1}+\overline{\boldsymbol{\omega}}^{T} \hat{\boldsymbol{\theta}}-\dot{y}_{d}+l^{1+2 \mu} z_{1}+\widehat{\psi}_{m} \tanh \left(\frac{z_{1}}{\delta_{1}}\right),
\end{gathered}
$$

where $N(k)$ is Nussbaum gain; $\widehat{\psi}_{m}$ is an estimate of $\psi_{m}$ with the estimation error $\widetilde{\psi}_{m}=\psi_{m}-\widehat{\psi}_{m} ; c_{1}$ is a positive constant. Using the inequality

$$
0 \leq\left|z_{1}\right|-z_{1} \tanh \left(\frac{z_{1}}{\delta}\right) \leq 0.2785 \delta
$$

where $\delta$ is a positive design parameter and substituting (35) and (36) into (34) yield

$$
\begin{aligned}
\dot{z}_{1}= & \kappa_{m}\left[z_{2}+N(k) \tau\right]+\Delta_{1}(y, t)+\widetilde{\boldsymbol{\theta}}^{T} \boldsymbol{\Gamma}\left(\tau_{1}-\dot{\hat{\boldsymbol{\theta}}}\right) \\
& +\sigma_{\theta} \widetilde{\boldsymbol{\theta}}^{T} \widehat{\boldsymbol{\theta}}+\tau-c_{1} z_{1}-l^{1+2 \mu} z_{1}-\widehat{\psi}_{m} \tanh \left(\frac{z_{1}}{\delta_{1}}\right),
\end{aligned}
$$

where $\tau_{1}=z_{1} \boldsymbol{\Gamma}^{-1} \overline{\boldsymbol{\omega}}-\sigma_{\theta}(\|\widehat{\boldsymbol{\theta}}\|) \boldsymbol{\Gamma}^{-1} \widehat{\boldsymbol{\theta}}$.

Define the quadratic function

$$
V_{1}=\frac{1}{2} z_{1}^{2}+\frac{1}{2} \widetilde{\boldsymbol{\theta}}^{T} \boldsymbol{\Gamma} \tilde{\boldsymbol{\theta}}+\frac{1}{2 r_{\psi}} \widetilde{\psi}_{m}^{2}
$$

From Young's inequality, we obtain

$$
z_{1} \tilde{x}_{2} \leq l^{1+2 \mu} z_{1}^{2}+\frac{l \bar{x}_{2}^{2}}{4}
$$


Differentiating $V_{1}$ with respect to time $t$ leads to

$$
\begin{aligned}
\dot{V}_{1}= & \kappa_{m} z_{1} z_{2}-c_{1} z_{1}^{2}+\kappa_{m} z_{1} N(k) \tau+\dot{k}+\frac{1}{4} l \bar{x}_{2}^{2} \\
& +\widetilde{\boldsymbol{\theta}}^{T} \boldsymbol{\Gamma}\left(\tau_{1}-\dot{\hat{\boldsymbol{\theta}}}\right)+\sigma_{\theta} \widetilde{\boldsymbol{\theta}}^{T} \widehat{\boldsymbol{\theta}}+\sigma_{\psi} \widetilde{\psi}_{m}^{T} \widehat{\psi}_{m} \\
& +\frac{1}{r_{\psi}} \widetilde{\psi}_{m}\left(r_{\psi} \tau_{1 \psi}-\dot{\hat{\psi}}_{m}\right)+0.2785 \delta_{1} \psi_{m},
\end{aligned}
$$

where $\tau_{1 \psi}=z_{1} \tanh \left(z_{1} / \delta_{1}\right)-\sigma_{\psi} \widehat{\psi}_{m}$.

Step 2. The time derivative of $z_{2}$ along with (31) is

$$
\dot{z}_{2}=v_{m, 3}-q_{2} l^{2} v_{m, 1}-\dot{\alpha}_{1}
$$

Define the function $\beta_{i}(2 \leq i \leq \rho)$ as

$$
\begin{aligned}
\beta_{i}= & \frac{\partial \alpha_{i-1}}{\partial y}\left(\boldsymbol{\omega}_{0}+\boldsymbol{\omega}^{T} \widehat{\boldsymbol{\theta}}\right)+\frac{\partial \alpha_{i-1}}{\partial \boldsymbol{\xi}_{0}} \dot{\boldsymbol{\xi}}_{0}+\frac{\partial \alpha_{i-1}}{\partial k} \dot{k}+\sum_{j=1}^{q} \frac{\partial \alpha_{i-1}}{\partial \boldsymbol{\xi}_{j}} \dot{\boldsymbol{\xi}}_{j} \\
& +\sum_{j=1}^{q} \sum_{i=1}^{m+1} \frac{\partial \alpha_{i-1}}{\partial \varsigma_{i, j}} \dot{\zeta}_{i, j}+\sum_{j=1}^{i} \frac{\partial \alpha_{i-1}}{\partial y_{r}^{(j-1)}} y_{r}^{(j)}+\sum_{j=1}^{m+1} \frac{\partial \alpha_{i-1}}{\partial \lambda_{j}} \dot{\lambda}_{j} .
\end{aligned}
$$

Define $z_{3}=v_{m, 3}-\alpha_{2}$ and $\alpha_{2}$ is chosen as

$$
\begin{gathered}
\alpha_{2}=-\widehat{\kappa}_{m} z_{1}-c_{2} z_{2}-l^{1+2 \mu}\left(\frac{\partial \alpha_{1}}{\partial y}\right)^{2} z_{2}-\beta_{2}+\frac{\partial \alpha_{1}}{\partial \widehat{\psi}_{m}} r_{\psi} \tau_{2 \psi} \\
+q_{2} l^{2} \nu_{m, 1}+\frac{\partial \alpha_{1}}{\partial \widehat{\boldsymbol{\theta}}} \boldsymbol{\Gamma} \tau_{2}-\widehat{\psi}_{m} \frac{\partial \alpha_{1}}{\partial y} \tanh \left(\frac{z_{2}\left(\partial \alpha_{1} / \partial y\right)}{\delta_{2}}\right) \\
\tau_{2}=\tau_{1}-\frac{\partial \alpha_{1}}{\partial y} \Gamma^{-1} \omega z_{2}+\Gamma^{-1}\left[0_{1 \times p}, z_{1} z_{2}, 0, \ldots, 0\right]^{T} \\
\tau_{2 \psi}=\tau_{1 \psi}+z_{2} \frac{\partial \alpha_{1}}{\partial y} \tanh \left(\frac{z_{2}\left(\partial \alpha_{1} / \partial y\right)}{\delta_{2}}\right)
\end{gathered}
$$

The time derivative of $z_{2}$ along with (41)-(43) is

$$
\begin{aligned}
\dot{z}_{2}= & z_{3}-\widehat{\kappa}_{m} z_{1}-c_{2} z_{2}-\frac{\partial \alpha_{1}}{\partial y} \widetilde{\boldsymbol{\theta}}^{T} \boldsymbol{\omega}-l^{1+2 \mu}\left(\frac{\partial \alpha_{1}}{\partial y}\right)^{2} z_{2} \\
& -\frac{\partial \alpha_{1}}{\partial y} \tilde{x}_{2}+\frac{\partial \alpha_{1}}{\partial \widehat{\boldsymbol{\theta}}}\left(\tau_{2}-\dot{\hat{\boldsymbol{\theta}}}\right)+\frac{\partial \alpha_{1}}{\partial \widehat{\psi}_{m}}\left(\tau_{2 \psi}-\dot{\hat{\psi}}_{m}\right) \\
& -\widehat{\psi}_{m} \frac{\partial \alpha_{1}}{\partial y} \tanh \left(\frac{z_{2}\left(\partial \alpha_{1} / \partial y\right)}{\delta_{2}}\right) .
\end{aligned}
$$

Consider the Lyapunov function candidate as

$$
V_{2}=V_{1}+\frac{1}{2} z_{2}^{2}
$$

The time derivative of $V_{2}$ along with (42) is

$$
\begin{aligned}
\dot{V}_{2} \leq & z_{2} z_{3}-c_{1} z_{1}^{2}-c_{2} z_{2}^{2}+\frac{1}{2} l \bar{x}_{2}^{2}+\widetilde{\boldsymbol{\theta}}^{T} \boldsymbol{\Gamma}\left(\tau_{1}-\dot{\hat{\boldsymbol{\theta}}}\right) \\
& +\kappa_{m} z_{1} N(k) \tau+\dot{k}+z_{2} \frac{\partial \alpha_{1}}{\partial \widehat{\boldsymbol{\theta}}}\left(\tau_{2}-\dot{\hat{\boldsymbol{\theta}}}\right)+\sigma_{\psi} \widetilde{\psi}_{m}^{T} \widehat{\psi}_{m} \\
& +\frac{1}{r_{\psi}} \widetilde{\psi}_{m}\left(r_{\psi} \tau_{1 \psi}-\dot{\hat{\psi}}_{m}\right)+\sigma_{\theta} \widetilde{\boldsymbol{\theta}}^{T} \widehat{\boldsymbol{\theta}}+z_{2} \frac{\partial \alpha_{1}}{\partial \widehat{\psi}_{m}}\left(\tau_{2 \psi}-\dot{\hat{\psi}}_{m}\right) \\
& +\sum_{j=1}^{2} 0.2785 \delta_{j} \psi_{m} .
\end{aligned}
$$

Step $i(3 \leq i \leq \rho-1)$. The time derivative of $z_{i}$ along with (31) is

$$
\dot{z}_{i}=v_{m, i}-q_{i} l^{i} v_{m, 1}-\dot{\alpha}_{i-1}
$$

Choose stabilizing function $\alpha_{i}$ as

$$
\begin{aligned}
& \alpha_{i}=-z_{i-1}-c_{i} z_{i}-l^{1+2 \mu}\left(\frac{\partial \alpha_{i-1}}{\partial y}\right)^{2} z_{i}+q_{i} l^{i} \nu_{m, 1}-\beta_{i} \\
& +\frac{\partial \alpha_{i-1}}{\partial \widehat{\boldsymbol{\theta}}} \boldsymbol{\Gamma} \tau_{i}+\frac{\partial \alpha_{i-1}}{\partial k} \dot{k}+\frac{\partial \alpha_{i-1}}{\partial \widehat{\psi}_{m}} r_{\psi} \tau_{i \psi}-\widehat{\psi}_{m} \frac{\partial \alpha_{i-1}}{\partial y} \\
& \times \tanh \left(\frac{z_{i}\left(\partial \alpha_{i-1} / \partial y\right)}{\delta_{i}}\right)-\left(\sum_{k=2}^{i-1} z_{k} \frac{\partial \alpha_{k-1}}{\partial \widehat{\boldsymbol{\theta}}}\right) \Gamma \frac{\partial \alpha_{i-1}}{\partial y} \boldsymbol{\omega} \\
& -\left(\sum_{k=2}^{i-1} z_{k} \frac{\partial \alpha_{k-1}}{\partial \widehat{\psi}_{m}}\right) \gamma_{\psi} \frac{\partial \alpha_{i-1}}{\partial y} \tanh \left(\frac{z_{i}\left(\partial \alpha_{i-1} / \partial y\right)}{\delta_{i}}\right) \\
& \tau_{i}=\tau_{i-1}-\frac{\partial \alpha_{i-1}}{\partial y} \Gamma^{-1} \boldsymbol{\omega} z_{i}, \\
& \tau_{i \psi}=\tau_{(i-1) \psi}+z_{i} \frac{\partial \alpha_{i-1}}{\partial y} \tanh \left(\frac{z_{i}\left(\partial \alpha_{i-1} / \partial y\right)}{\delta_{i}}\right) .
\end{aligned}
$$

Consider the Lyapunov function candidate as

$$
V_{i}=V_{i-1}+\frac{1}{2} z_{i}^{2}
$$

The time derivative of $V_{i}$ along with (47) and (48) is

$$
\begin{aligned}
\dot{V}_{i} \leq & z_{i} z_{i+1}-\sum_{j=1}^{i} c_{j} z_{j}^{2}+\frac{i}{4} l \bar{x}_{2}^{2}+\sum_{j=2}^{i} z_{j} \frac{\partial \alpha_{j-1}}{\partial \widehat{\boldsymbol{\theta}}}\left(\tau_{j}-\dot{\hat{\boldsymbol{\theta}}}\right) \\
& +\widetilde{\boldsymbol{\theta}}^{T} \Gamma\left(\tau_{i}-\dot{\hat{\boldsymbol{\theta}}}\right)+\sum_{j=2}^{i} z_{j} \frac{\partial \alpha_{j-1}}{\partial \widehat{\psi}_{m}}\left(\tau_{j \psi}-\dot{\hat{\psi}}_{m}\right)+\sigma_{\psi} \widetilde{\psi}_{m}^{T} \widehat{\psi}_{m}
\end{aligned}
$$




$$
\begin{aligned}
& +\frac{1}{r_{\psi}} \widetilde{\psi}_{m}\left(r_{\psi} \tau_{1 \psi}-\dot{\hat{\psi}}_{m}\right)-\sum_{j=3}^{i} \sum_{k=2}^{j-1} z_{j} \frac{\partial \alpha_{j-1}}{\partial \widehat{\boldsymbol{\theta}}} z_{k} \frac{\partial \alpha_{k-1}}{\partial y} \boldsymbol{\Gamma}^{-1} \boldsymbol{\omega} \\
& -\sum_{j=3}^{i} \sum_{k=2}^{j-1} z_{j} \frac{\partial \alpha_{j-1}}{\partial \widehat{\psi}_{m}} z_{k} \frac{\partial \alpha_{k-1}}{\partial y} \tanh \left(\frac{z_{j}\left(\partial \alpha_{j-1} / \partial y\right)}{\delta_{j}}\right) \\
& +\sigma_{\theta} \tilde{\boldsymbol{\theta}}^{T} \widehat{\boldsymbol{\theta}}+\kappa_{m} z_{1} N(k) \tau+\dot{k}+\sum_{j=1}^{i} 0.2785 \delta_{j} \psi_{m} .
\end{aligned}
$$

Define $z_{\rho}=v_{m, \rho}-\alpha_{\rho-1}$ and the time derivative of $z_{\rho}$ along with (31) is

$$
\dot{z}_{\rho}=u^{*}+v_{m, \rho+1}-q_{\rho} l^{\rho} v_{m, 1}-\dot{\alpha}_{\rho-1} .
$$

Finally, the actual control signal designed as

$$
u^{*}=\alpha_{\rho}-v_{m, \rho+1} \text {. }
$$

Choose the tuning functions and parameter adaptation law as

$$
\begin{gathered}
\dot{\hat{\boldsymbol{\theta}}}=\tau_{\rho}, \\
\dot{\hat{\psi}}_{m}=r_{\psi} \tau_{\rho \psi} .
\end{gathered}
$$

To prepare for the stability analysis, a candidate Lyapunov function for the closed-loop system is chosen as

$$
V_{\rho}=V_{\rho-1}+\frac{1}{2} z_{\rho}^{2}+V_{x}
$$

The time derivative of $V_{\rho}$ along with (50) and (51) is

$$
\begin{aligned}
\dot{V}_{\rho} \leq & -\sum_{i=1}^{\rho} c_{j} z_{j}^{2}-\frac{1}{2} l\|\overline{\mathbf{x}}\|^{2}+\kappa_{m} z_{1} N(k) \tau+\dot{k} \\
& +\frac{\rho}{4} l\left\|\bar{x}_{2}\right\|^{2}-\frac{\sigma_{10}}{2} \widetilde{\boldsymbol{\theta}}^{T} \widetilde{\boldsymbol{\theta}}-\frac{\sigma_{\psi}}{2} \widetilde{\psi}_{m}^{2}+\frac{\sigma_{\psi}}{2} \psi_{m}^{2} \\
& +\left\|\mathbf{P} \mathbf{L}^{-1}\right\|^{2} \psi_{m}^{2}+\frac{13}{2} \sigma_{10} M_{1}^{2}+\sum_{i=1}^{\rho} 0.2785 \delta_{i} \psi_{m} \\
\leq & -C_{0} V+D+\kappa_{m} z_{1} N(k) \tau+\dot{k},
\end{aligned}
$$

where

$$
\begin{gathered}
C_{0}=\min \left\{c_{1}, \ldots, c_{\rho}, \frac{\sigma_{10}}{\lambda_{\max }(\boldsymbol{\Gamma})}, \frac{\sigma_{\psi}}{2}, \frac{l}{\lambda_{\max }(\mathbf{P})}\right\}, \\
D=\left[\frac{13}{2} \sigma_{10} M_{1}^{2}+\frac{\sigma_{\psi}}{2} \psi_{m}^{2}+\frac{\rho}{4} l\left\|\bar{x}_{2}\right\|^{2}\right. \\
\left.+\sum_{i=1}^{\rho} 0.2785 \delta_{i} \psi_{m}+\left\|\mathbf{P L}^{-1}\right\|^{2} \psi_{m}^{2}\right] .
\end{gathered}
$$

Multiplying (55) by $\exp \left(C_{0} t\right)$ yields and integrating (55) over $[0, t]$, we have

$$
\begin{aligned}
V_{\rho} \leq & V_{\rho}(0) e^{-C_{0} t}+e^{-C_{0} t} \int_{0}^{t}\left[\kappa_{m} N(k)+1\right] \dot{k}^{C_{0} \tau} d \tau \\
& +\int_{0}^{t} D e^{-C_{0}(t-\tau)} d \tau .
\end{aligned}
$$

Next, at time $t, p_{1}$ actuator failures occur, which results in an abrupt change of $\boldsymbol{\theta}$, owing to the change of values of these parameters is finite. Moreover, from (28) and (30), we have

$$
\int_{0}^{t} D e^{-C_{0}(t-\tau)} d \tau
$$

which is bounded on $[0, t]$. Let $C_{d}$ and $C_{N}$ be the upper bound of $\int_{0}^{t} D e^{-C_{0}(t-\tau)} d \tau$ and $e^{-C_{0} t} \int_{0}^{t}\left[\kappa_{m} N(k)+1\right] \dot{k} e^{C_{0} \tau} d \tau$

$$
\begin{gathered}
C_{d}=\sup _{t \in[0, t]}\left(\int_{0}^{t} D e^{-C_{0}(t-\tau)} d \tau\right), \\
C_{N}=\sup _{t \in[0, t]}\left(e^{-C_{0} t} \int_{0}^{t}\left[\kappa_{m} N(k)+1\right] \dot{k} e^{C_{0} \tau} d \tau\right) .
\end{gathered}
$$

From (58) and (59), we have

$$
\begin{aligned}
0 \leq & V_{\rho}(t) \leq V_{\rho}(0)+\frac{C_{d}}{C_{0}} \\
& +e^{-C_{0} t} \int_{0}^{t}\left[\kappa_{m} N(k)+1\right] \dot{k}^{C_{0} \tau} d \tau .
\end{aligned}
$$

According to Lemma 4, we have $V_{\rho}(t), k(t)$ and $\int_{0}^{t} \kappa_{m} N(k) \dot{k} d \tau$ bound on $[0, t)$. Therefore, $z_{i}(t), \ldots, z_{\rho}, N(k)$ are bound on $[0, t)$ for all $t>0$, and all signals in the closedloop system are bounded on $[0, t)$ for all $t>0$. According to the discussion in [37], we see that the above conclusion is true for $t=+\infty$. Thus, we know that $x_{i}, z_{i}$ are semiglobally uniformly ultimately bounded, we also have inequalities (61) as well as

$$
\left|z_{1}\right| \leq \sqrt{2\left(V_{\rho}(0)-\frac{C_{d}}{C_{0}}\right) e^{-C_{0} t}+2\left(\frac{C_{d}}{C_{0}}+C_{N}\right)}
$$

Choosing appropriate positive matrix $\Gamma$ such that $\lambda_{\text {min }}(\Gamma)>0$. Furthermore, in order to achieve the tracking error convergent to a small neighborhood around zero, the parameters $c_{i}, \sigma_{\psi}$ and $\Gamma$ should be chosen appropriately to make $C_{N}$ as small as desired. In this sense, we have guaranteed transient response. This result of transient response of the system is a direct consequence of the underlying robust filter structure of the ARFTC controller.

\section{Application Example}

To demonstrate the effectiveness of the proposed approach, we consider the following nonlinear system:

$$
\begin{gathered}
\dot{x}_{1}=x_{2}+f_{1}(y)+a_{1} y+a_{2} \sin (y)+\Delta_{1}(x, t), \\
\dot{x}_{2}=a_{3} y^{2}+a_{4} y+y x_{2}+f_{2}(y)+b_{01} \beta_{1}(y) u_{1} \\
+b_{02} \beta_{2}(y) u_{2}+\Delta_{2}(x, t), \\
y=x_{1},
\end{gathered}
$$

where $a_{1}=12, a_{2}=-1, a_{3}=1, a_{4}=-1, b_{01}=1$, $b_{02}=0.3, \beta_{1}(y)=\beta_{2}(y)=1+|y \cos (y)|, f_{1}(y)=3 x_{2} y^{2}$, 


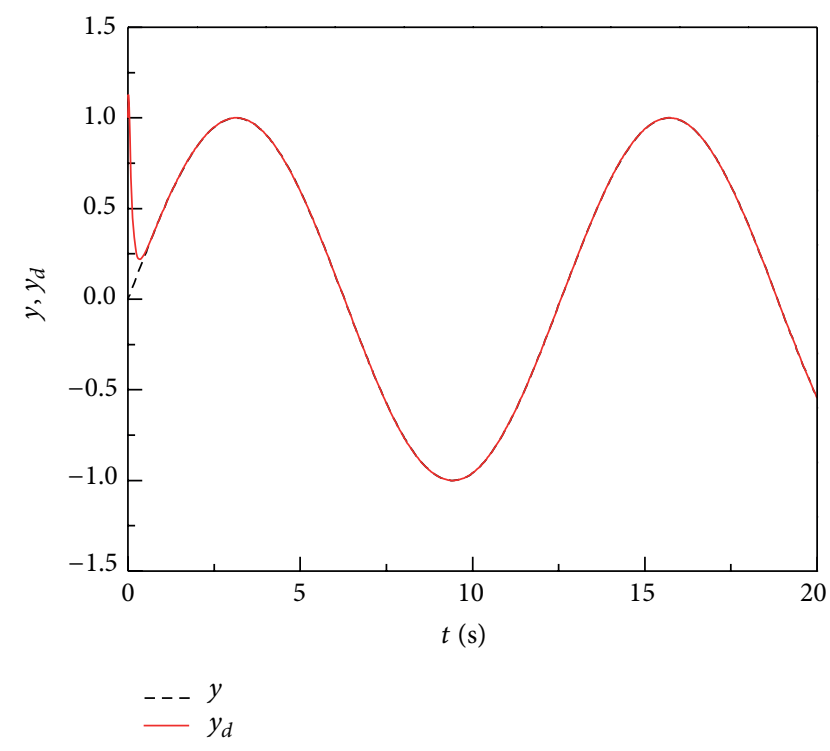

FIGURE 1: Plant output and reference signal.

and $f_{2}(y)=y^{2}$. In the simulation, RBFNNs are applied and we select the centers and widths as neural networks $\widehat{\mathbf{W}}_{1}^{T} \boldsymbol{\Phi}_{1}(y)$ contain 25 nodes (i.e., $\left.l_{1}=25\right)$ with centers $c_{l}\left(l=1,2, \ldots, l_{1}\right)$ evenly spaced in $[-4.0,4.0] \times[-4.0,4.0]$ and width centers $\eta_{l}=1.0\left(l=1,2, \ldots, l_{1}\right)$, and 25 nodes (i.e., $\left.l_{2}=25\right)$ with centers $c_{l}\left(l=1,2, \ldots, l_{2}\right)$ spaced in $[-4.0,4.0] \times[-4.0,4.0] \times$ $[-4.0,4.0] \times[-4.0,4.0]$ and widths $\eta_{l}=1.0\left(l=1,2, \ldots, l_{2}\right)$ for $\mathrm{NN} \widehat{\mathbf{W}}_{2}^{T} \boldsymbol{\Phi}_{2}(y)$.

Note that the same reduced order model was used by Tang et al. [19] and thus will provide a platform to compare the robust adaptive control (RAC) based fault-tolerant control (FTC) with the adaptive robust fault-tolerant control (ARFTC) schemes.

Case 1 (no failures occur). The simulation results are shown in Figures 1 and 2 for $y_{d}=\sin (0.5 t)$. In the first set of simulations (see Figure 1), all unstructured modeling errors and disturbances are assumed to be zero; that is, $\Delta_{i}=0$ for $i=1,2$. The initial conditions $x=\left[\begin{array}{ll}0 & 1\end{array}\right]^{T}$. The simulation parameters are as follows:

$$
\begin{aligned}
& \Gamma=\operatorname{diag}\left\{\begin{array}{lllllllllll}
0.5 & 0.5 & 10 & 10 & 5 & 5 & 1 & 1 & 1 & 1 & 1
\end{array}\right\}, \\
& \mathbf{q}=\left[\begin{array}{ll}
1 & 1
\end{array}\right]^{T}, \quad r_{\psi}=1.5, \\
& \mu=1, \quad \kappa=0.5, \quad \delta_{1}=1, \quad \delta_{2}=0.5, \\
& c_{1}=12, \quad c_{2}=5, \quad k(0)=0, \quad \gamma(y)=1.6 y^{2}, \\
& \widehat{\theta}(0)=\operatorname{diag}\left\{\begin{array}{lllllllllll}
0 & 0 & 0 & 0 & 0 & 0 & 5 & -0.4 & 0.4 & -0.5 & 0.5
\end{array}\right\} \text {, } \\
& N(k)=k^{2} \cos (k) \text {. }
\end{aligned}
$$

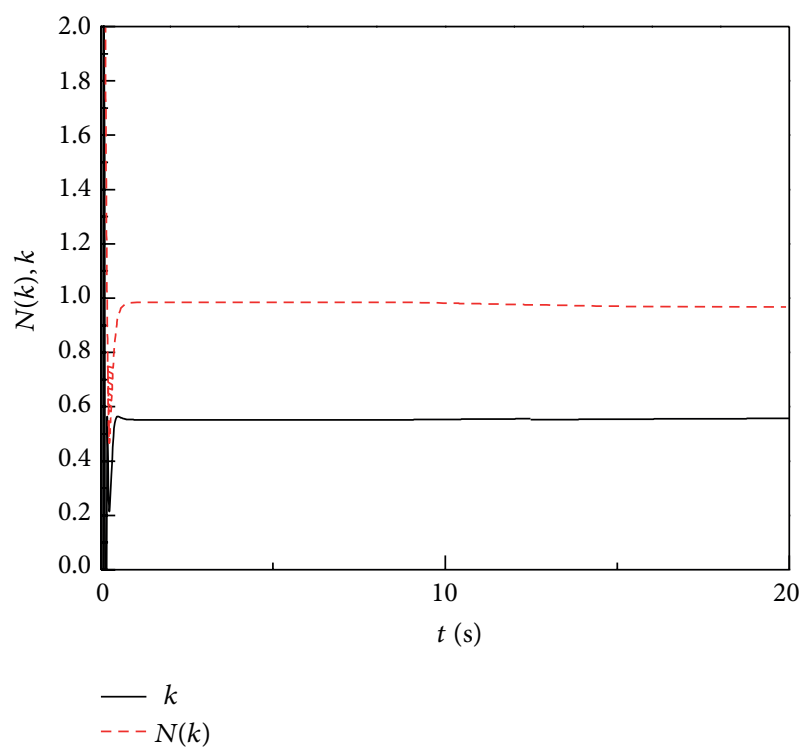

FIGURE 2: Nussbaum gain $N(k)$ and its argument $k$.

Case 2. Actuator faults occur and the actual control signal is given by

$$
\begin{gathered}
u_{1}(t)= \begin{cases}v_{1}(t), & t \in(0,30) \\
\bar{u}_{1}+\bar{d}_{11} f_{11}(t), & t \in(30, \infty),\end{cases} \\
u_{2}(t)=v_{2}(t), \quad t \in(0, \infty) .
\end{gathered}
$$

Failure parameters are chosen to be $\bar{u}_{1}=6, d_{11}=2, f_{11}(t)=$ $0.5 \sin (t)$.

The reference command is chosen as $y_{d}(t)=\sin (0.5 t)$. Details of RAC based FTC can be obtained from [19]. Additionally, the controller parameters were chosen such that the control input profiles would be comparable for both schemes. Two cases are considered in order to illustrate the effectiveness of the proposed scheme.

In the first set of simulations, $\Delta i=0$ for $i=1,2$. Then, from Figures 1 and 2, we conclude that the presented control scheme can work effectively for nonlinear systems without failures in actuators. Furthermore, from Figure 3, both the systems perform well initially and have similar control input profiles. With the actuator failure, which causes a bigger jump in the parameter value, the tracking error stays close to zero in ARFTC based scheme but deviates significantly in the RAC based scheme. This can be explained as follows. The design of the robust component of the ARFTC control law has already incorporated such jumps in parameter values, and hence, it is better suited to handle the parametric uncertainties introduced due to actuator failures.

The second set of simulations (see Figure 4) is performed where disturbances were introduced to test the performance of the two schemes in presence of unknown bounded uncertainties. We set $\Delta_{1}=0.5 \sin (3 t)$ and $\Delta_{2}=0.3 \sin (5 t)$. The parametric uncertainty and unstructured uncertainty bounds are incorporated in the design of the baseline robust controller in ARFTC, resulting in guaranteeing desired transient 


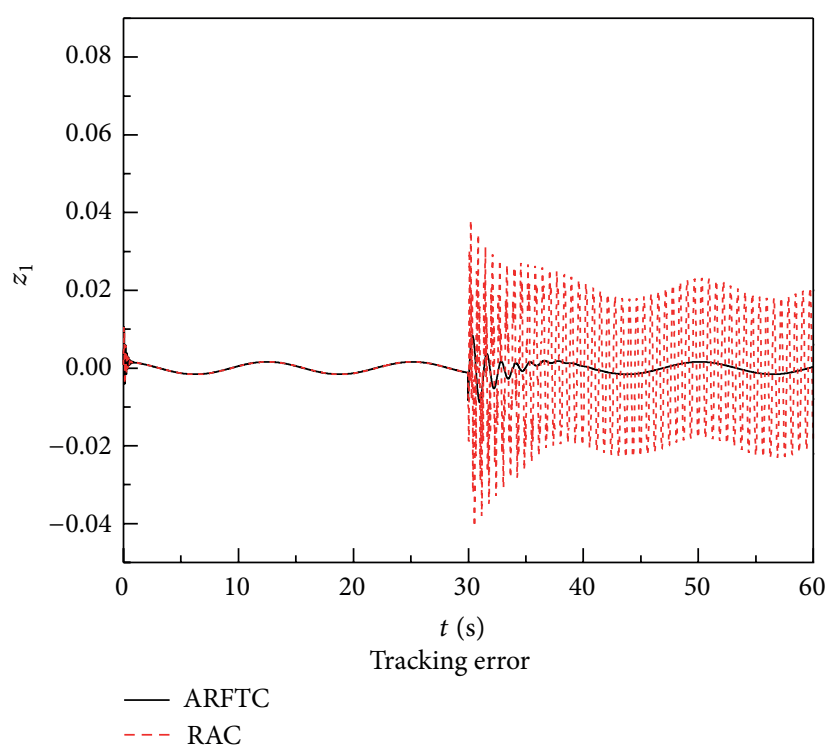

(a)

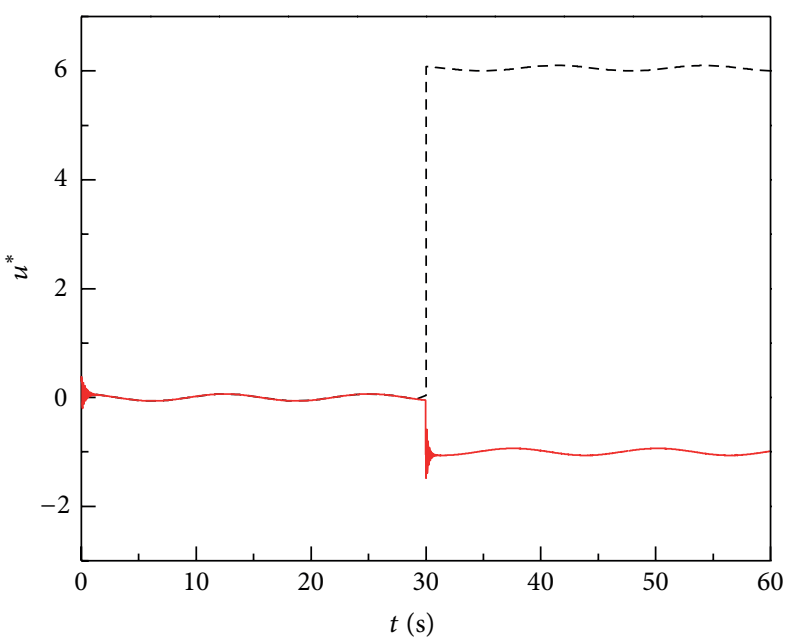

Control signals: ARFTC $---u_{1}$

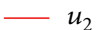

(b)

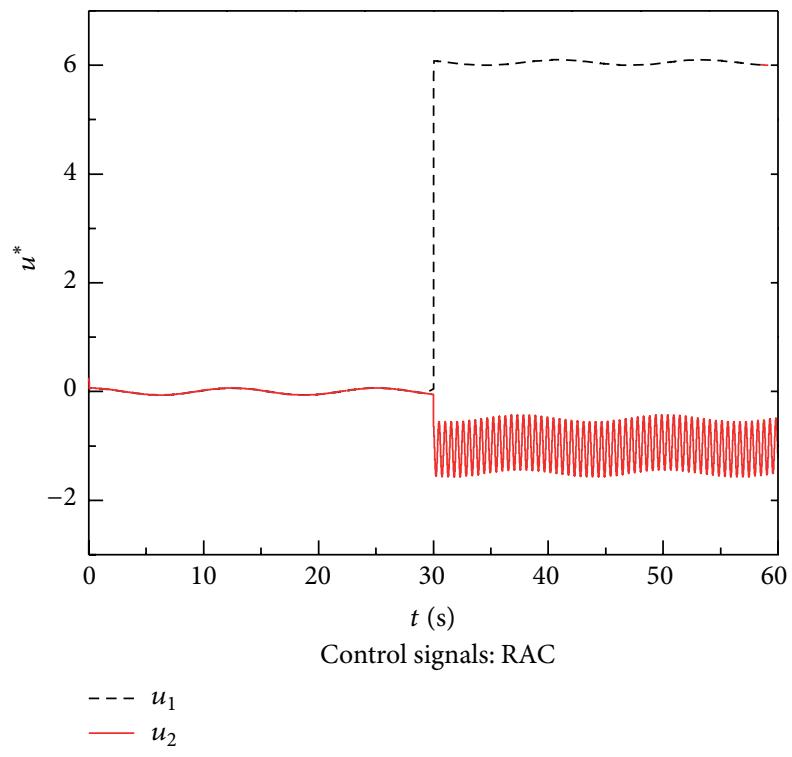

(c)

FIGURE 3: Tracking error and control signals for ARFTC versus RAC based fault-tolerant schemes.

response and acceptable steady-state tracking error. The performance of the RAC based scheme deteriorates significantly in presence of unstructured modeling uncertainties, which are inherent in any realistic system model. Therefore, the achievable performance using the proposed scheme is superior to that of RAC based schemes.

\section{Conclusions}

In this paper, an adaptive actuator failure compensation scheme based on the robust fault-tolerant control approach has been proposed. A linearly parameterized model with unknown parameters and actuator failure parameters has been established. The Nussbaum gain approach has been exploited to relax the assumption on the control gain signs. The control scheme introduced switching $\sigma$ algorithms to ensure that the estimation of time-varying parameters is bounded. The designed controller does not require precise information of failures, thereby improving the engineering application value. Finally, simulation studies have shown that the proposed adaptive robust control law is effective.

\section{Conflict of Interests}

The authors declare that there is no conflict of interests regarding the publication of this paper. 


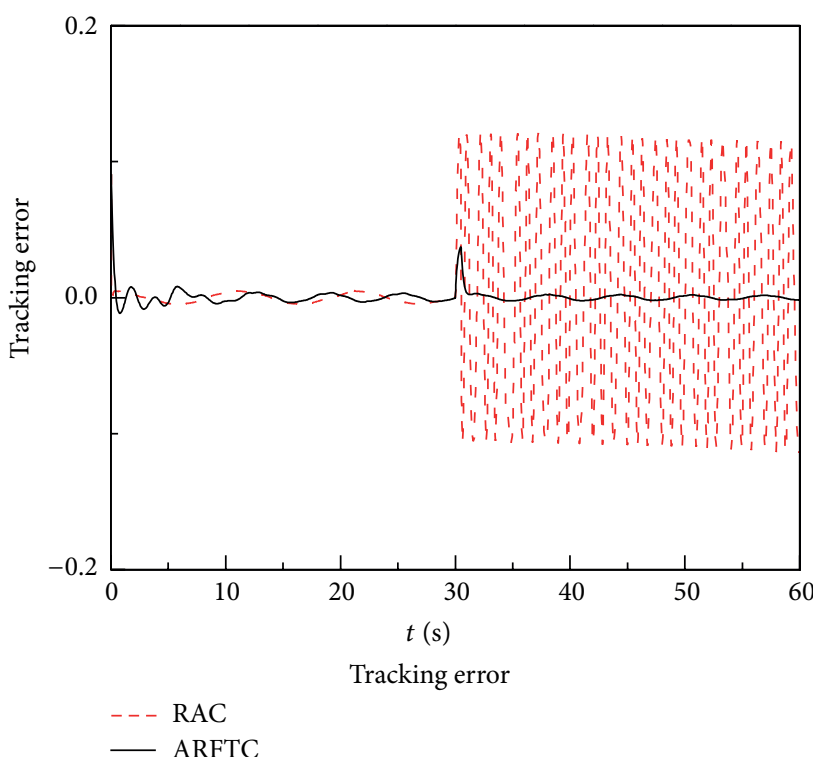

(a)

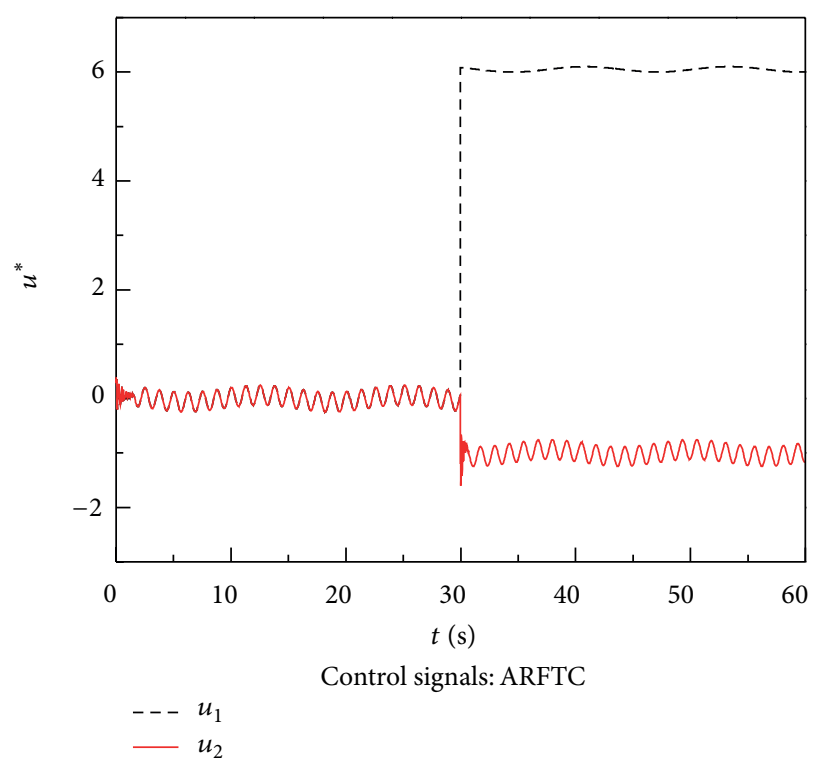

(b)

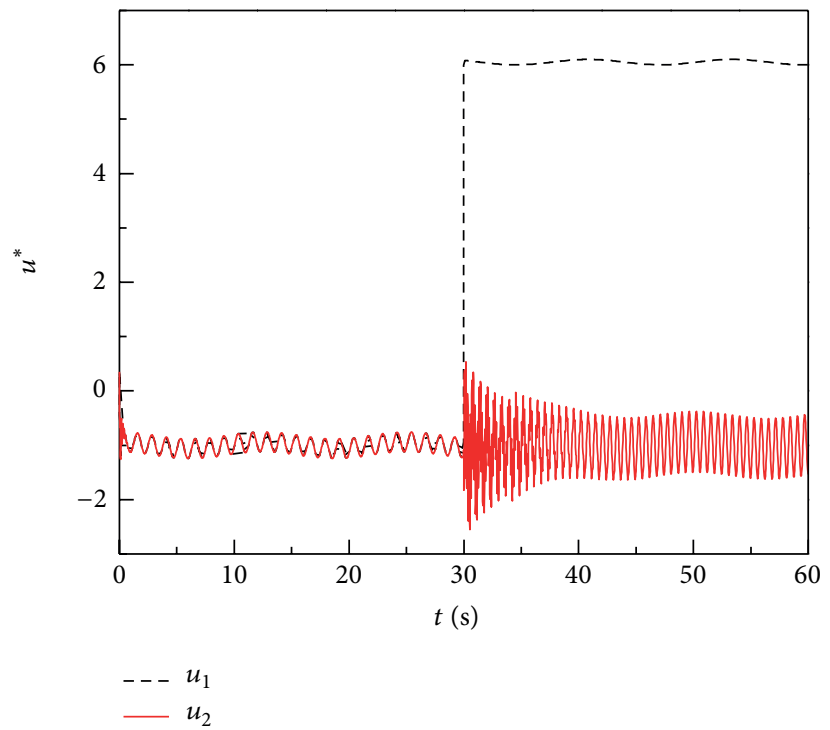

(c)

FIGURE 4: Tracking error and control signals for ARFTC versus RAC based fault-tolerant schemes in presence of disturbances.

\section{Acknowledgments}

This work was supported in part by the National Natural Science Foundation of China under Grant no. 51305203, in part by the Specialized Research Fund for the Doctoral Program of Higher Education (Ministry of Education of China) under Grant no. 20133219120026, and in part by the Jiangsu Planned Projects for Postdoctoral Research Funds under Grant no. 1302002A.

\section{References}

[1] I. Kanellakopoulos, P. V. Kokotovic, and A. S. Morse, "Systematic design of adaptive controllers for feedback linearizable systems," IEEE Transactions on Automatic Control, vol. 36, no. 11, pp. 1241-1253, 1991.

[2] M. M. Polycarpou and A. J. Helmicki, "Automated fault detection and accommodation: a learning systems approach," IEEE Transactions on Systems, Man and Cybernetics, vol. 25, no. 11, pp. 1447-1458, 1995.

[3] A. T. Venuri and M. M. Polycarpon, "On-line approximation methods for robust fault detection," in Proceedings of the 13th IFAC World Congress, pp. 319-324, San Francisco, Calif, USA, 1996.

[4] I. Hwang, S. Kim, Y. Kim, and C. E. Seah, "A survey of fault detection, isolation, and reconfiguration methods," IEEE Transactions on Control Systems Technology, vol. 18, no. 3, pp. 636653, 2010. 
[5] P. Garimella and B. Yao, "An adaptive robust framework for model-based state fault detection," in Proceedings of the American Control Conference, pp. 5692-5697, Minneapolis, Minn, USA, June 2006.

[6] B. Jiang, M. Staroswiecki, and V. Cocquempot, "Fault accommodation for nonlinear dynamic systems," IEEE Transactions on Automatic Control, vol. 51, no. 9, pp. 1578-1583, 2006.

[7] H. Wang and S. Daley, "Actuator fault diagnosis: an adaptive observer-based technique," IEEE Transactions on Automatic Control, vol. 41, no. 7, pp. 1073-1078, 1996.

[8] D. Yue, J. Lam, and D. W. C. Ho, "Reliable $H_{\infty}$ control of uncertain descriptor systems with multiple time delays," IEE Proceedings: Control Theory and Applications, vol. 150, no. 6, pp. 557-564, 2003.

[9] D. Ye and G.-H. Yang, "Adaptive reliable $H_{\infty}$ control for linear time-delay systems via memory state feedback," IET Control Theory and Applications, vol. 1, no. 3, pp. 713-721, 2007.

[10] G. Tao, S. Chen, and S. M. Joshi, "An adaptive actuator failure compensation controller using output feedback," IEEE Transactions on Automatic Control, vol. 47, no. 3, pp. 506-511, 2002.

[11] X. Tang, G. Tao, and S. M. Joshi, "Adaptive actuator failure compensation for nonlinear MIMO systems with an aircraft control application," Automatica, vol. 43, no. 11, pp. 1869-1883, 2007.

[12] X. Tang, G. Tao, and S. M. Joshi, "Adaptive actuator failure compensation for parametric strict feedback systems and an aircraft application," Automatica, vol. 39, no. 11, pp. 1975-1982, 2003.

[13] J. Yao, Z. Jiao, and D. Ma, "Extended-state-observer-based output feedback nonlinear robust control of hydraulic systems with backstepping," IEEE Transactions on Industrial Electronics, 2014.

[14] J. Yao, G. Yang, Z. Jiao, and D. Ma, "Adaptive robust motion control of direct-drive dc motors with continuous friction compensation," Abstract and Applied Analysis, vol. 2013, Article ID 837548, 14 pages, 2013.

[15] J. Yao, Z. Jiao, and D. Ma, "Adaptive robust control of DC motors with extended state observer," IEEE Transactions on Industrial Electronics, vol. 61, no. 7, pp. 3630-3637, 2014.

[16] A. Datta and P. A. Ioannou, "Performance analysis and improvement in model reference adaptive control," IEEE Transactions on Automatic Control, vol. 39, no. 12, pp. 2370-2387, 1994.

[17] M. Corradini, G. Orlando, and G. Parlangeli, "A fault tolerant sliding mode controller for accommodating actuator failures," in Proceedings of the 44th IEEE Conference on Decision and Control, European Control Conference, pp. 3091-3096, 2005.

[18] D. Kim and Y. Kim, "Robust variable structure controller design for fault tolerant flight control," Journal of Guidance, Control, and Dynamics, vol. 23, no. 3, pp. 430-437, 2000.

[19] X. Tang, G. Tao, and S. M. Joshi, "Adaptive output feedback actuator failure compensation for a class of non-linear systems," International Journal of Adaptive Control and Signal Processing, vol. 19, no. 6, pp. 419-444, 2005.

[20] Z. Gao and S. X. Ding, "Actuator fault robust estimation and fault-tolerant control for a class of nonlinear descriptor systems," Automatica, vol. 43, no. 5, pp. 912-920, 2007.

[21] J. Yao, Z. Jiao, and D. Ma, "High accuracy tracking control of hydraulic rotary actuators with modelling uncertainties," IEEE/ASME Transactions on Mechatronics, vol. 19, no. 2, pp. 633-641, 2014.
[22] W. Sun, H. Gao Sr., and O. Kaynak, "Finite frequency $H_{\infty}$ control for vehicle active suspension systems," IEEE Transactions on Control Systems Technology, vol. 19, no. 2, pp. 416-422, 2011.

[23] W. Sun, Y. Zhao, J. Li, L. Zhang, and H. Gao, "Active suspension control with frequency band constraints and actuator input delay," IEEE Transactions on Industrial Electronics, vol. 59, no. 1, pp. 530-537, 2012.

[24] X. Tang, G. Tao, and S. M. Joshi, "Adaptive actuator failure compensation control of parametric strict-feedback systems with zero dynamics," in Proceedings of the 40th IEEE Conference on Decision and Control (CDC '01), pp. 2031-2036, December 2001.

[25] X. Tang, G. Tao, and S. M. Joshi, "An adaptive control scheme for output feedback nonlinear systems with actuator failures," in Proceedings of the 15th IFAC World Congress, 2002.

[26] S. Gayaka and B. Yao, "Output feedback based adaptive robust fault-tolerant control for a class of uncertain nonlinear systems," Journal of Systems Engineering and Electronics, vol. 22, no. 1, pp. 38-51, 2011.

[27] Z. Zhang, S. Xu, Y. Guo, and Y. Chu, "Robust adaptive outputfeedback control for a class of nonlinear systems with timevarying actuator faults," International Journal of Adaptive Control and Signal Processing, vol. 24, no. 9, pp. 743-759, 2010.

[28] R. D. Nussbaum, "Some remarks on a conjecture in parameter adaptive control," Systems and Control Letters, vol. 3, no. 5, pp. 243-246, 1983.

[29] D. R. Mudgett and A. S. Morse, "Adaptive stabilization of linear systems with unknown high-frequency gains," IEEE Transactions on Automatic Control, vol. 30, no. 6, pp. 549-554, 1985.

[30] Z. T. Ding, "Adaptive control of nonlinear systems with unknown virtual control coefficients," International Journal of Adaptive Control and Signal Processing, vol. 14, no. 5, pp. 505$517,2000$.

[31] Z. Ding and X. Ye, "A flat-zone modification for robust adaptive control of nonlinear output feedback systems with unknown high-frequency gains," IEEE Transactions on Automatic Control, vol. 47, no. 2, pp. 358-363, 2002.

[32] X. Ye, "Adaptive nonlinear output-feedback control with unknown high-frequency gain sign," IEEE Transactions on Automatic Control, vol. 46, no. 1, pp. 112-115, 2001.

[33] Y. Xudong and J. Jingping, "Adaptive nonlinear design without a priori knowledge of control directions," IEEE Transactions on Automatic Control, vol. 43, no. 11, pp. 1617-1621, 1998.

[34] S. S. Ge and J. Wang, "Robust adaptive neural control for a class of perturbed strict feedback nonlinear systems," IEEE Transactions on Neural Networks, vol. 13, no. 6, pp. 1409-1419, 2002.

[35] S. S. Ge, F. Hong, and T. H. Lee, "Adaptive neural control of nonlinear time-delay systems with unknown virtual control coefficients," IEEE Transactions on Systems, Man, and Cybernetics, Part B, vol. 34, no. 1, pp. 499-516, 2004.

[36] Y. Zhang and P. A. Ioannou, "Robustness and performance of a modified adaptive backstepping controller," International Journal of Adaptive Control and Signal Processing, vol. 12, no. 3, pp. 247-265, 1998.

[37] E. P. Ryan, "A universal adaptive stabilizer for a class of nonlinear systems," Systems and Control Letters, vol. 16, no. 3, pp. 209-218, 1991. 


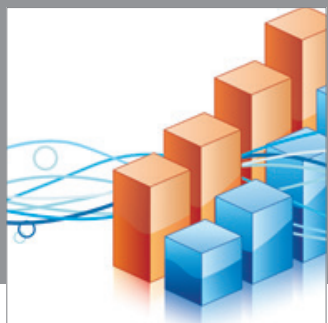

Advances in

Operations Research

mansans

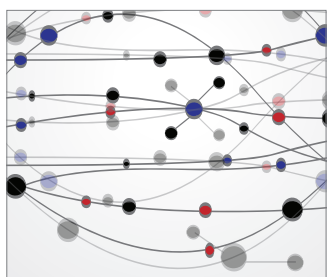

The Scientific World Journal
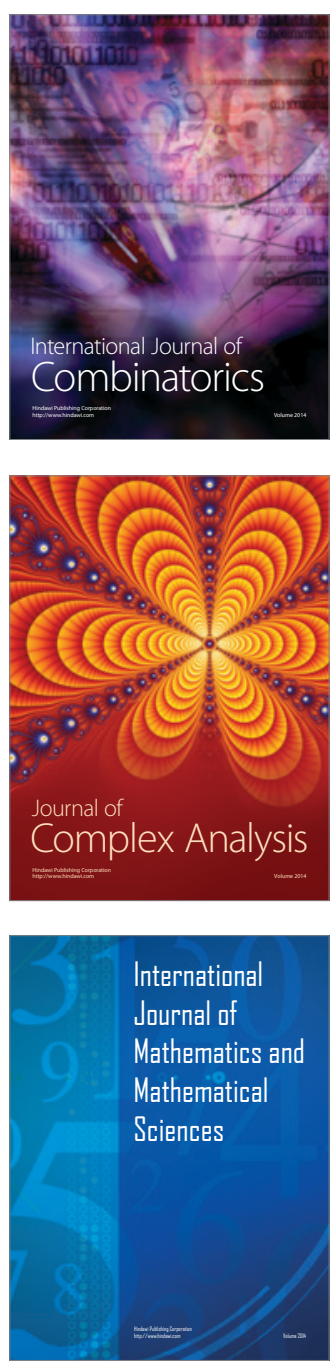
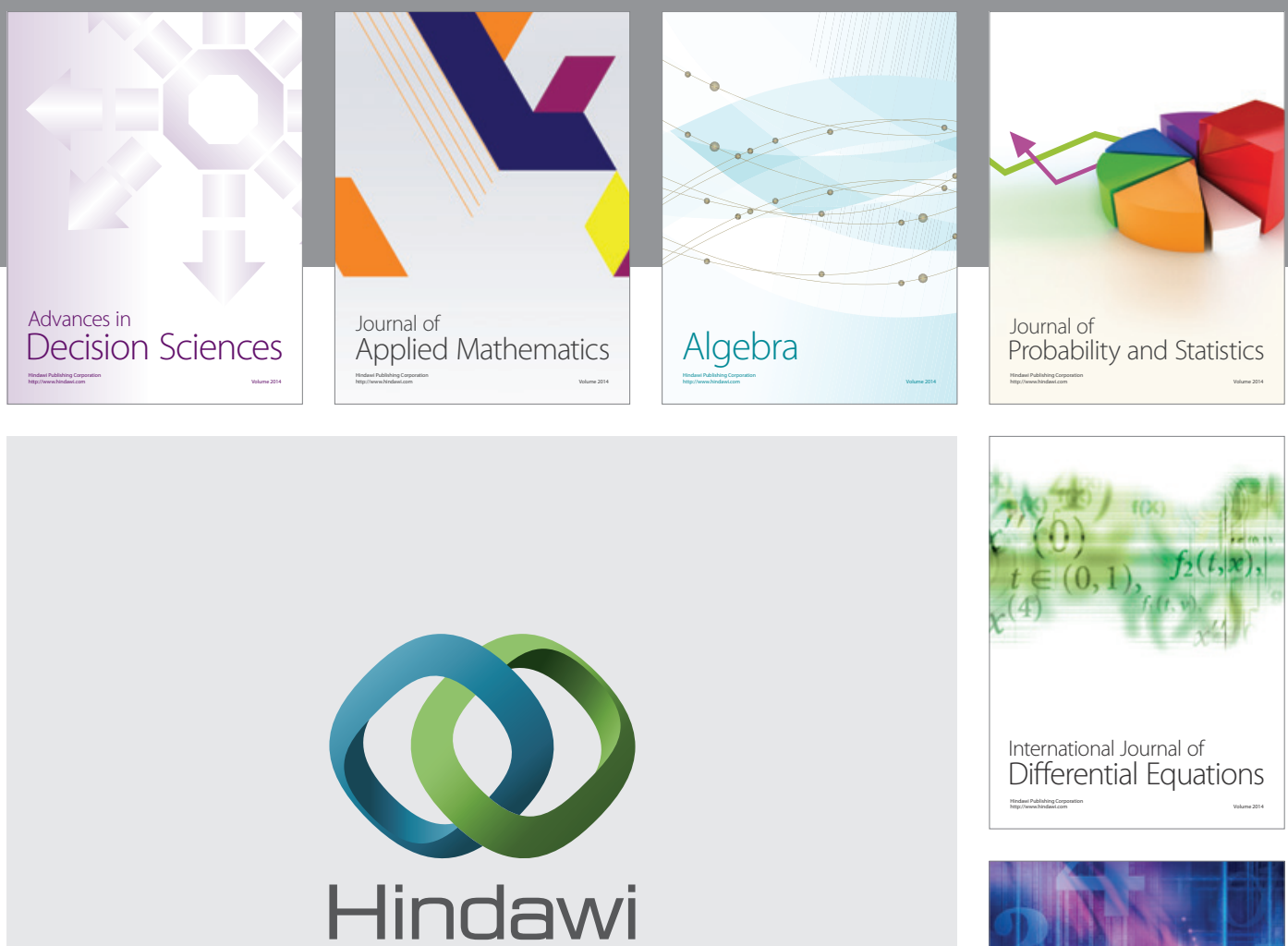

Submit your manuscripts at http://www.hindawi.com
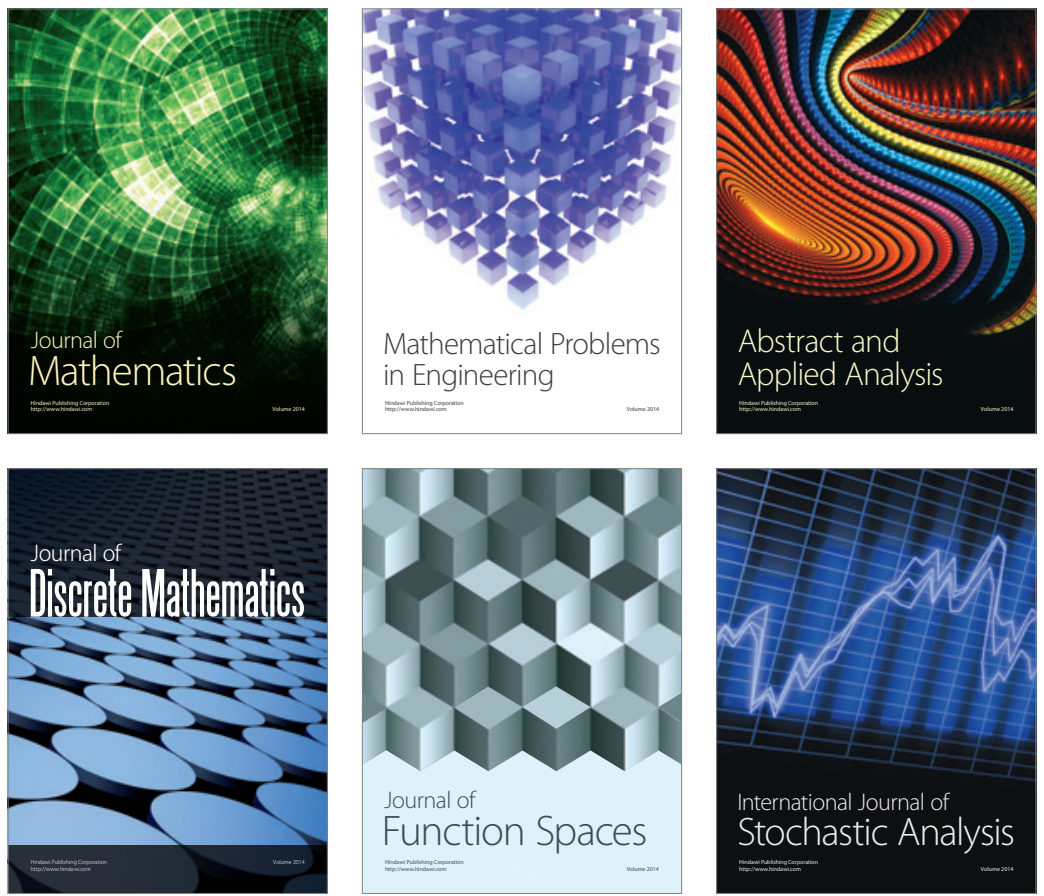

Journal of

Function Spaces

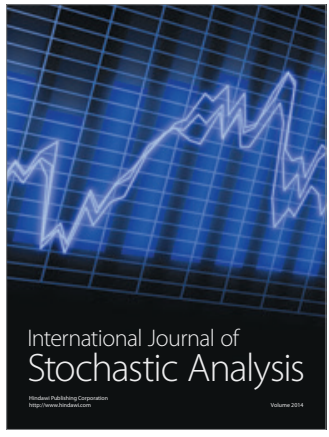

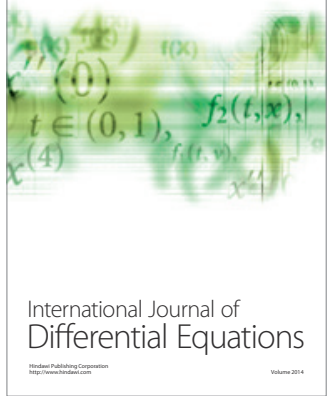
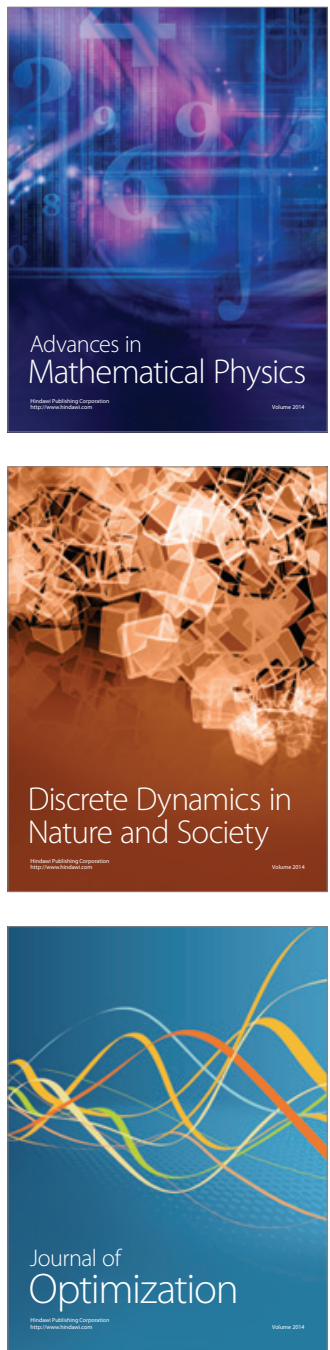